\title{
Interferometric science results on young stellar objects
}

\author{
F. Malbet \\ Laboratoire d'Astrophysique de Grenoble, \\ Université J. Fourier/CNRS, BP 53, F-38041 Grenoble cedex 9, France
}

\begin{abstract}
Long-baseline interferometry at infrared wavelengths allows the innermost regions around young stars to be observed. These observations directly probe the location of the dust and gas in the disks. The characteristic sizes of these regions found are larger than previously thought. These results have motivated in part a new class of models of the inner disk structure, but the precise understanding of the origin of these low visibilities is still in debate. Mid-infrared observations probe disk emission over a larger range of scales revealing mineralogy gradients in the disk. Recent spectrally resolved observations allow the dust and gas to be studied separately showing that the Brackett gamma emission can find its origin either in a wind or in a magnetosphere and that there is probably no correlation between the location of the Brackett gamma emission and accretion. In a certain number of cases, the very high spatial resolution reveals very close companions and can determine their masses. Overall, these results provide essential information on the structure and the physical properties of close regions surrounding young stars especially where planet formation is suspected to occur.
\end{abstract}

Key words:

accretion disks; stars: pre-main-sequence; stars: emission-line; stars: mass loss; stars: winds, outflows; planetary systems: protoplanetary disks; infrared: stars; techniques: interferometric; techniques: spectroscopic

\section{Introduction}

Many physical phenomena occur in the inner regions of the disks which surround young stars. The matter which eventually falls onto the stellar surface works its way through an accreting circumstellar disk which is subject to turbulence, convection, external and internal irradiation. The disks, which are rotating in a quasi-Keplerian motion, are probably the birth location of future planetary systems. Strong outflows, winds and even jets find their origin in the innermost regions of many young stellar systems. The mechanisms of these ejection processes are not well understood but they are probably connected to accretion. Most stars

Email address: Fabien.Malbet@obs.ujf-grenoble.fr (F. Malbet) 
are born in multiple systems which can be very tightly bound and therefore have a strong impact on the physics of the disk inner regions.

The details of all these physical processes are not well understood yet because of lack of data to constrain them. The range of physical parameters which define best the inner disk regions in young stellar objects are:

- radius ranging from $0.1 \mathrm{AU}$ to $10 \mathrm{AU}$

- temperature ranging from $150 \mathrm{~K}$ to $4000 \mathrm{~K}$

- velocity ranging from $10 \mathrm{~km} / \mathrm{s}$ to a few $100 \mathrm{~km} / \mathrm{s}$

The instrumental requirements to investigate the physical conditions in such regions are therefore driven by the spectral coverage which must encompass the near and mid infrared from 1 to $20 \mu \mathrm{m}$. Depending on the distance of the object (typically between $75 \mathrm{pc}$ and $450 \mathrm{pc}$ ) the spatial resolution required to probe the inner parts of disks ranges between fractions and a few tens of milli-arcseconds. Since the angular resolution of astronomical instruments depends linearly on the wavelength and inversely on the telescope diameter, observing in the near and mid infrared wavelength domain points toward telescopes of sizes ranging from ten to several hundreds of meters. The only technique that allows such spatial resolution is therefore infrared interferometry.

Millan-Gabet et al. [39], published in Protostars and Planets $V$, reviewed the main results obtained with infrared interferometry in the domain of young stars between 1998 and 2005. I presented a review talk on this subject at the IAU Symposium 243 on Star-disk interaction in young stars [34] as well as R. Akeson in several schools in 2008 [3, 4].

The purpose of the present review is to give the latest results in this field. Section 2 briefly explains the principles of infrared interferometry and lists the literature on the observations carried out with this technique. Section 3 focuses on the main results obtained on disk physics (sizes, structures, dust and gas components,...) and Sect. 4 presents results on other phenomena constrained by interferometry (winds, magnetosphereic accretion, multiple systems,...). In Sect. 5, I finish the review with the type of results that can be expected in the future.

\section{Infrared interferometry}

\subsection{Principle and observations}

Long baseline optical interferometry consists in mixing the light received from an astronomical source and collected by several independent telescopes separated from each other by tens or even hundreds of meters. The light beams are then overlapped and form an interference pattern if the optical path difference between the different arms of the interferometer - taking into account paths from the source up to the detector - is smaller than the coherence length of the incident wave (typically of the order of several microns). This interference figure is composed of fringes, i.e. a succession of stripes of faint (destructive interferences) and bright (constructive interferences) intensity. By measuring the contrast 

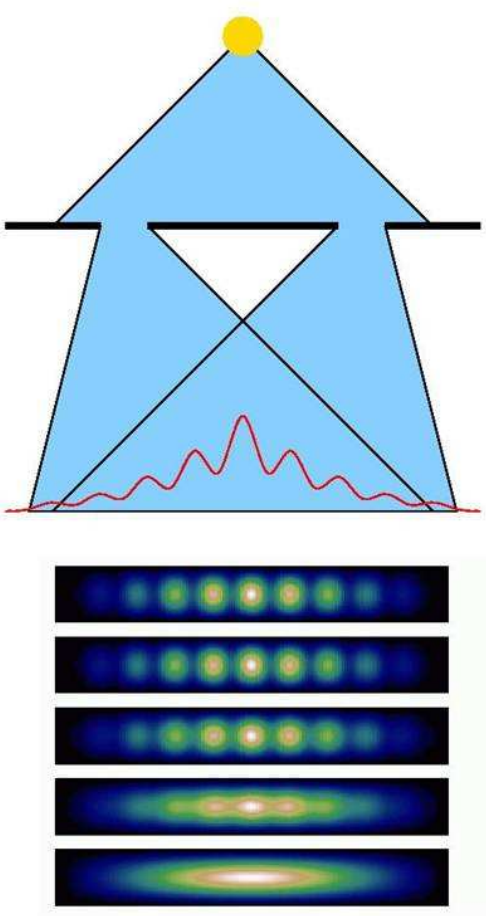
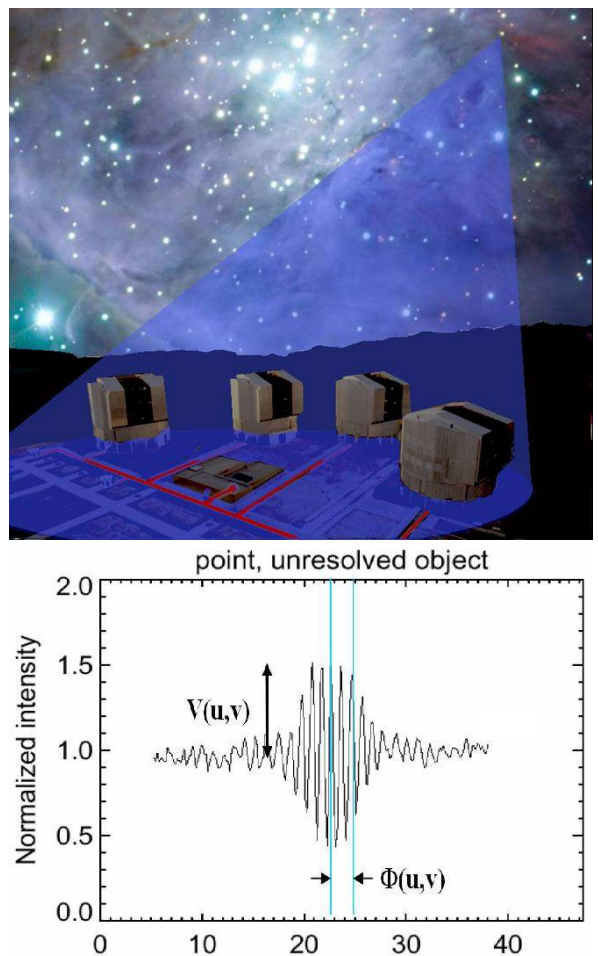

Figure 1: Principle of interferometry. Upper panels: Young's slit experiment (left) compared to optical interferometry (right): in both cases light travels from a source to a plane where the incoming wavefront is split. The telescope apertures play the same role as the Young's slits. The difference lies in the propagation of light after the plane. In the case of optical interferometry, the instrument controls the propagation of light down to the detectors. At the detector plane, the light beams coming from the two apertures are overlapped. Lower panels: interference fringes whose contrast changes with the morphology of the source. Left panel shows fringes whose contrast varies from 0 to 1 . Right panel displays actual stellar fringes but scanned along the optical path. The measure of the complex visibilities corresponds to the amplitude of the fringes for the visibility amplitude and the position of the fringes in wavelength units for the visibility phase.

of these fringes, i.e. the normalized flux difference between the darkest and brightest regions, information about the morphology of the observed astronomical source can be recovered. Figure 1 illustrates this principle.

\subsection{Instruments available for inner regions studies}

Interferometric observations of young stellar objects were and are still performed at six different facilities on seven different instruments (see Table 1). We can classify these observations into three different categories:

- Small-aperture interferometers: PTI, IOTA and ISI were the first facilities to be operational for YSO observations in the late 1990's (see Figs. 2 and 3). They have provided mainly the capability of measuring visibility amplitudes and lately closure phases. The latest one, CHARA, has an aperture diameter of $1 \mathrm{~m}$. The instruments 
Table 1: Interferometers involved in YSO science

\begin{tabular}{llllll}
\hline \hline Facility & $\begin{array}{l}\text { Instrument } \\
\text { observable }\end{array}$ & $\begin{array}{l}\text { Wavelength } \\
\text { (microns) }\end{array}$ & $\begin{array}{l}\text { Numbers of } \\
\text { apertures }\end{array}$ & $\begin{array}{l}\text { Aperture } \\
\text { diameter }(\mathrm{m})\end{array}$ & $\begin{array}{l}\text { Baseline } \\
(\mathrm{m})\end{array}$ \\
\hline \hline PTI & $V^{2}$ & $H, K$ & 3 & 0.4 & $80-110$ \\
\hline IOTA & $V^{2}, \mathrm{CP}$ & $H, K$ & 3 & 0.4 & $5-38$ \\
\hline ISI & heterodyne & 11 & $2(3)$ & 1.65 & $4-70$ \\
\hline KI & $V^{2}$, nulling & $K$ & 2 & 10 & 80 \\
\hline VLTI/AMBER & $V^{2}, \mathrm{CP}$ & $1-2.5$ & $3(8)$ & $8.2 / 1.8$ & $40-130$ \\
& $($ imaging $)$ & $/$ spectral & & & $/ 8-200$ \\
\hline VLTI/MIDI & $V^{2}(/ \mathrm{CP})$ & $8-13$ & $2(4)$ & $8.2 / 1.8$ & $40-130$ \\
& & $/$ spectral & & & $5-200$ \\
\hline CHARA & $V^{2}, \mathrm{CP}$ & $1-2.5$ & $2 / 4(6)$ & 1 & $6-350$ \\
& $($ imaging $)$ & /spectral & & & \\
\hline LBT & imaging, & $1-10$ & 2 & 8.4 & \\
\hline \hline
\end{tabular}

$V^{2}$ : visibility measurement; CP: closure phase.

Acronyms. PTI: Palomar Testbed Interferometer; IOTA: Infrared and Optical Telescope Array (closed since 2006); ISI: Infrared Spatial Interferometer; KI: Keck Interferometer; VLTI: Very Large Telescope Interferometer; CHARA: Center for High Angular Resolution Array; LBT: Large Binocular Telescope (not yet operational).

are mainly accessible through team collaboration. IOTA was shut down in 2007 and PTI in January 2009.

- Large-aperture interferometers: KI, VLTI and soon LBT are facilities with apertures larger than $8 \mathrm{~m}$. The instruments are widely open to the astronomical community through general calls for proposals. Lately, these facilities have significantly increased the number of young objects observed.

- Instruments with spectral resolution: CHARA, MIDI and AMBER provide spectral resolution from a few hundred up to 10,000 whereas other instruments mainly provided broadband observations. The spectral resolution allows the various phenomena occurring in the environment of young stars to be separated.

\subsection{Elements of bibliography}

Figure 2 displays the number of published results, and show that it is increasing with time and improved facilities. At the date of the conference there were 38 refereed articles 
YSO refereed results (1998-2008)

12

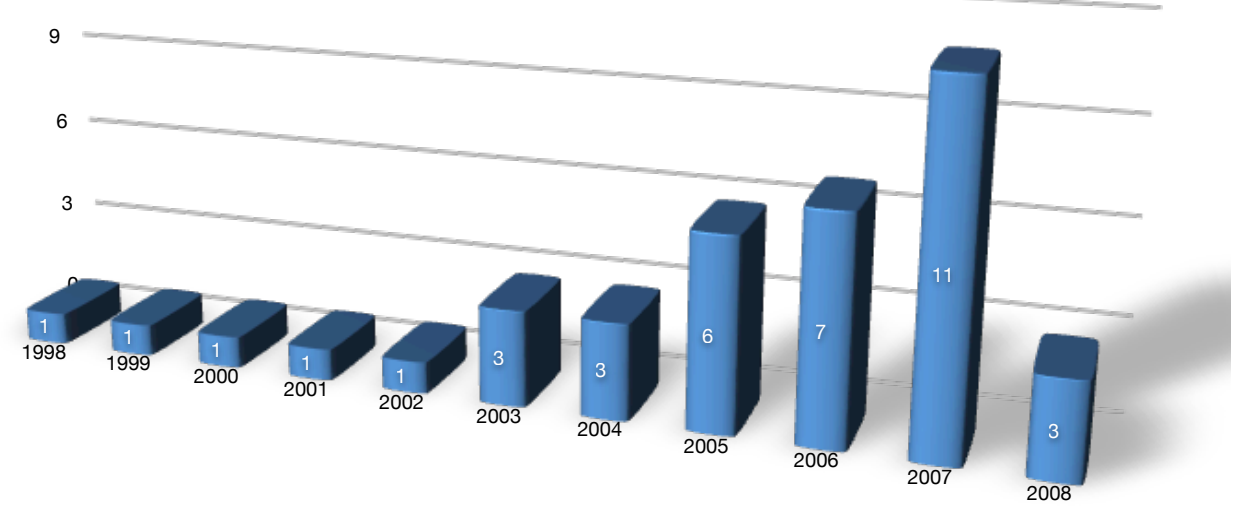

YSO number of observations

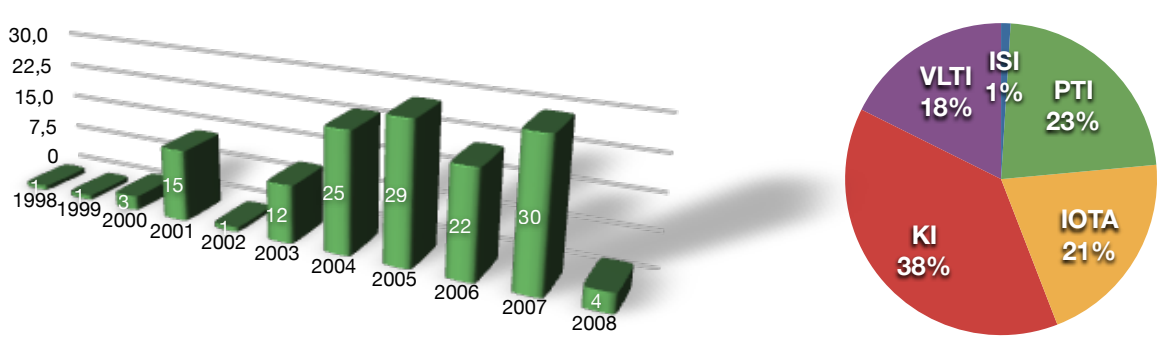

Figure 2: Young stellar objects observed by interferometry and number of refereed papers published in the period 1998-2008 (top graph). The statistics of the year 2008 is not complete. The bottom left graph presents the number of YSO targets observed in the same period and the right bottom one gives the distribution by interferometer.

published in the field of young stars corresponding to 76 young stellar objects observed 1 .

Graphs in Fig. 3 show that the distribution of observed object is rather well distributed among the various facilities. Several categories of young stellar systems have been observed at milli-arcsecond scales mainly in the near-infrared wavelength domain, but also in the mid-infrared one. They include the brightest Herbig Ae/Be stars, the fainter T Tauri stars and the few FU Orionis. Finally most observations were carried out in broad band but the advent of large aperture interferometers like the VLTI and KI allow higher spectral resolution to be obtained.

\footnotetext{
${ }^{1}$ See the references by chronological order: $[36,43,6,42,60,20,12,64,32,21,61,38,7,18,46,9,5,16$, 40, 49, 1, 41, 44, 50, 17, 35, 57, 27, 31, 15, 51, 19, 10, 13, 33, 29, 56, 26, 2. .
} 

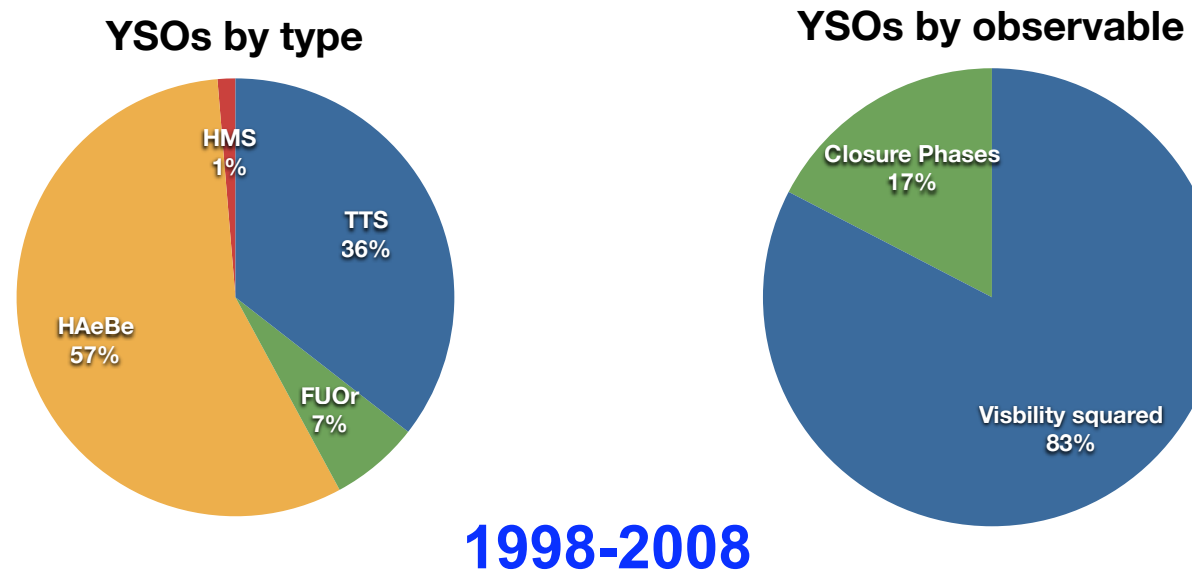

YSOs by spectral resolution

YSOs by spectral band
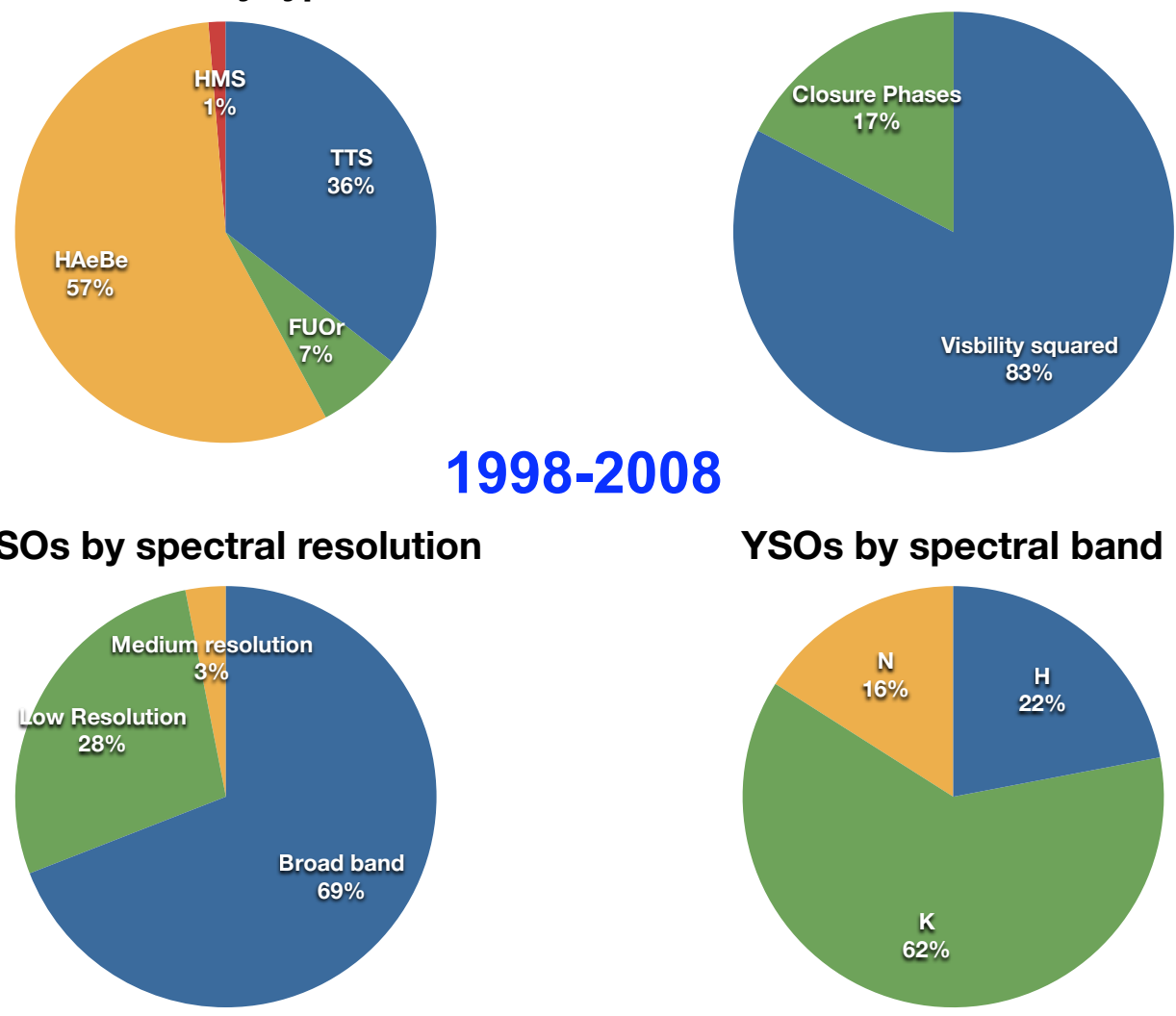

Figure 3: Young stellar objects observed by interferometry in the period 1998-2008. Upper left: distribution by YSO type. Upper right: distribution by type of observable. Lower left: distribution by spectral resolution. Lower right: distribution by wavelength of observation. The statistics are the same as the one of Fig. 2 .

\section{Inner disk physics}

Most YSO studies are focused on the physics of the inner regions of disks. They started with the determination of rough sizes of emitting regions and naturally led to more constraints on the disk structure. Spectrally resolved mid-infrared observations are able to identify different types of dust grains. Spectrally resolved near-infrared observations permit to spatially discriminate between gas and dust.

\subsection{Sizes of circumstellar structures}

Disks are known to be present around young stars. Some ten years ago, disks were believed to behave "normally" with a radial temperature distribution following a power-law $T \propto r^{-q}$ with $q$ ranging between 0.5 and 0.75 . The value of $q$ depends on the relative effect of irradiation from the central star in comparison with heat dissipation due to accretion. This model was successful to reproduce ultraviolet and infrared excesses in spectral energy distributions (SEDs). Malbet and Bertout [37] investigated the potential of optical long baseline interferometry to study the disks of T Tauri stars and FU Orionis stars. They 
found that the structure would be marginally resolved but observations would be possible with baselines of the order of $100 \mathrm{~m}$ with a visibility amplitude remaining high.

First observations of the brighter Herbig Ae/Be stars showed that the observed visibilities were much smaller than expected especially for those objects where the accretion plays a little role. Monnier and Millan-Gabet [45] pointed out that the interferometric sizes of these objects were much larger than expected from the standard disk model. They plotted the sizes obtained as function of the stellar luminosity and found that there was a strong correlation following a $L^{0.5}$ law over two decades. This behavior is consistent with the variation of radius of dust sublimation with respect to the central star luminosity: the dust distribution radius shifts to larger radii for more luminous objects because the temperature is larger than the sublimation limit $(\sim 1000-1500 \mathrm{~K})$. Only the most massive Herbig Be stars seem to be compliant with the standard accretion disk model.

In the meantime, in order to account for the near-infrared characteristics of SEDs and in particular a flux excess around $\lambda=3 \mu \mathrm{m}$, Natta et al. 48] proposed that disks around Herbig Ae/Be stars have an optically thin inner cavity and create a puffed-up inner wall of optically thick dust at the dust sublimation radius. More realistic models were developed afterward which take more physical properties into account [14, 47, 25]. However as pointed out by Vinković et al. [62] and recently by Schegerer et al. [52] on the specific and actual case of RY Tau, the disk models are not the only ones that can reproduce the measurements: models with a disk halo or envelope can also match the data.

Observations at KI [12, 18, 7] found also large NIR sizes for lower-luminosity T Tauri stars, in many cases even larger than would be expected from extrapolation of the HAe relation. It is interpreted by the fact that the accretion disk contributes significantly to the luminosity emitted by the central region and therefore this additional luminosity must be taken into account in the relationship. However in these systems uncertainties are still large and very few measurements per object have been obtained. In order to interpret all the T Tauri measurements, Akeson et al. [7] need to introduce a new physical component like optically thick gas emission in the inner hole and extended structure around the objects.

Characteristic dimensions of the emitting regions at $10 \mu \mathrm{m}$ were found by [32] to be ranging from $1 \mathrm{AU}$ to $10 \mathrm{AU}$. The sizes of their sample stars correlate with the slope of the $10-25 \mu \mathrm{m}$ infrared spectrum: the reddest objects are the largest ones. Such a correlation is consistent with a different geometry in terms of flaring or flat (self-shadowed) disks for sources with strong or moderate mid-infrared excess, respectively, demonstrating the power of interferometry not only to probe characteristic sizes of disks but also to derive information on the vertical disk structure.

\subsection{Constraints on disk structure}

Theoreticians start discussing slightly different scenarios of the inner regions around young stars. For example, the inner puffed-up wall is modeled with a curved shape by Isella and Natta [25] due to the very large vertical density gradient and the dependence of grain evaporation temperature on gas density as expected when a constant evaporation temperature is assumed. Tannirkulam et al. [55] proposed that the geometry of the rim depends on the composition and spatial distribution of dust due to grain growth and settling. 
Vinković and Jurkić 63. presented a model-independent method of comparison of NIR visibility data of YSOs. The method based on scaling the measured baseline with the YSO distance and luminosity removes the dependence of visibility on these two variables. They found that low luminosity Herbig Ae stars are best explained by the uniform brightness ring and the halo model, T Tauri stars with the halo model, and high luminosity Herbig Be stars with the accretion disk model, but they admit that the validity of each model is not well established.

At the moment, only two objects have been thoroughly studied: FU Orionis [36, 38, 50, and MWC 147 [29]. FU Ori has been observed on 42 nights over a period of 6 years from 1998 to 2003 with 287 independent measurements of the fringe visibility at 6 different baselines ranging from 20 to $110 \mathrm{~m}$ in length, in the $H$ and $K$ bands. The data not only resolves FU Ori at the AU scale, but also allows the accretion disk scenario to be tested. The most probable interpretation is that FU Ori hosts an active accretion disk whose temperature law is consistent with the standard model. In the mid infrared, Quanz et al. [50] resolved structures that are also best explained with an optically thick accretion disk. A simple accretion disk model fits the observed SED and visibilities reasonably well and does not require the presence of any additional structure such as a dusty envelope. However Zhu et al. [65] revisited this issue using detailed radiative transfer calculations to model the recent, high signal-to-noise ratio data obtained on FU Ori from the IRS instrument on the Spitzer Space Telescope. They found that a physically plausible flared disk irradiated by the central accretion disk matches the observations. Their accretion disk model with outer disk irradiation by the inner disk reproduces the spectral energy distribution and their model is consistent with near-infrared interferometry, but there remains some inconsistencies with mid-infrared interferometric results. This is why one should remain careful with results coming from surveys having only few measurements per object.

Millan-Gabet et al. [40] obtained $K$-band observations of three other FU Orionis objects, V1057 Cyg, V151 Cyg, and Z CMa-SE and found that all three objects appear significantly more resolved than expected from simple models of accretion disks tuned to fit the SEDs. They believe that emission at the scale of tens of AU in the interferometer field of view is responsible for the low visibilities, originating in scattering by large envelopes surrounding these objects. Li Causi et al. [33] have measured again interferometric visibilities of Z CMa with VLTI/AMBER.

Kraus et al. 29] have shown that they are able to derive the temperature radial distribution of the disk around MWC 147 from the interferometric measurements using the spectral variation of the visibilities in low resolution with AMBER and MIDI (see Fig. 4). A similar work has been attempted at PTI with larger uncertainties [17].

On the pure theoretical side, very few physical models achieved to fit interferometric data simultaneously with SEDs. Using a two-layer accretion disk model, Lachaume et al. [30] found satisfactory fits for SU Aur, in solutions that are characterized by the midplane temperature being dominated by accretion, while the emerging flux is dominated by reprocessed stellar photons. Since the midplane temperature drives the vertical structure of the disk, there is a direct impact on the measured visibilities that are not necessarily taken into account by other models. In the MIR range, Schegerer et al. [52] was able to fit both the 

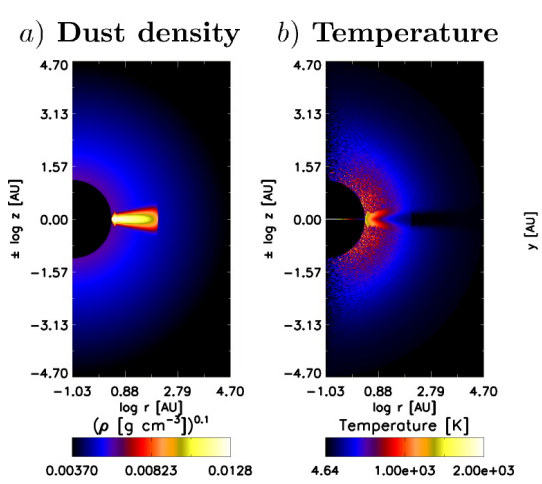

c) Image $K$-band
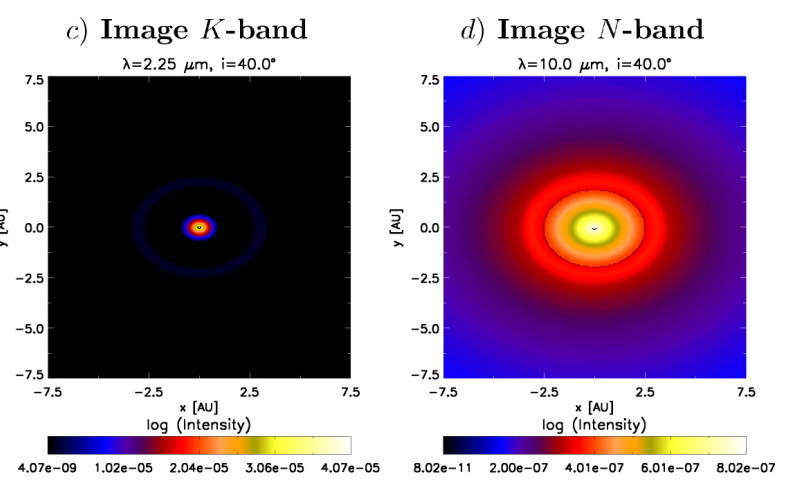

e) SED (different incl.)

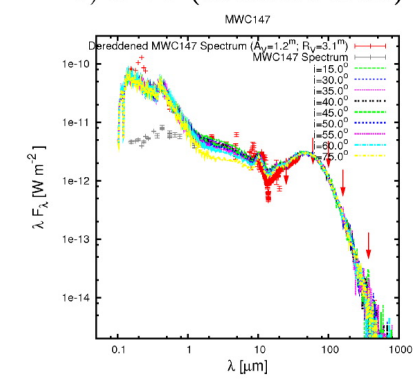

g) Visibilities NIR (IOTA/PTI)

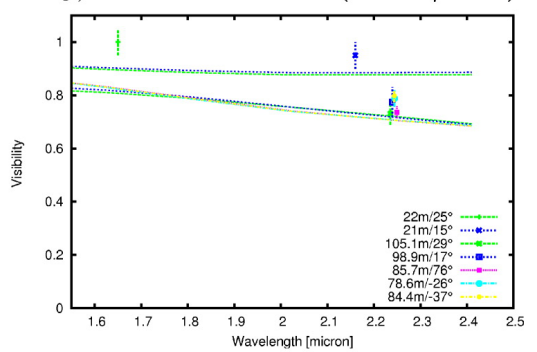

i) Visibilities MIR (MIDI)

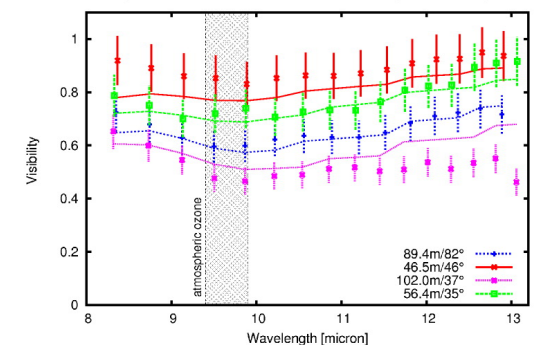

f) SED (best-fit incl. $i=60^{\circ}$ )

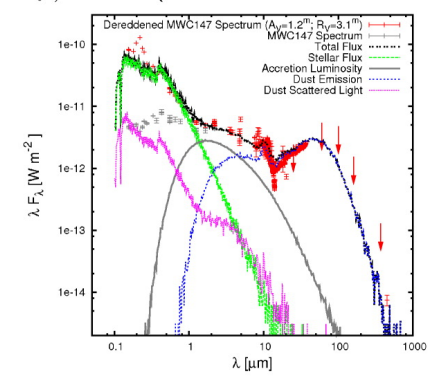

h) Visibilities NIR (AMBER)
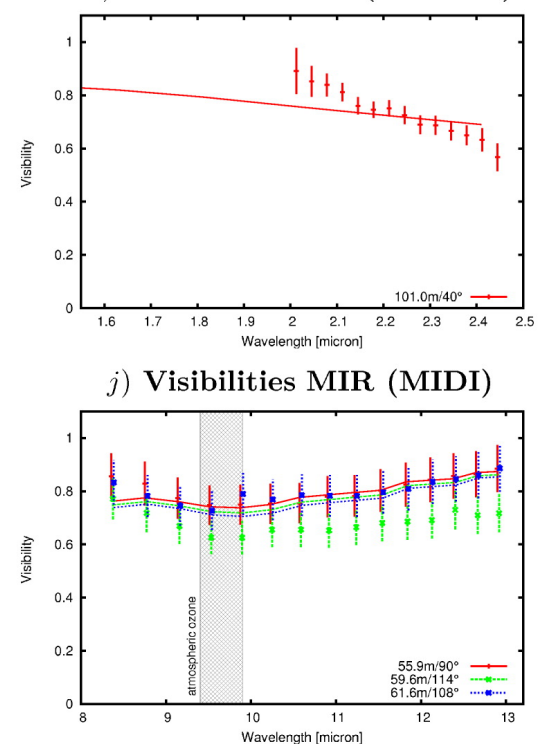

j) Visibilities MIR (MIDI)

Figure 4: Radiative transfer model constrained by interferometric measurements on MWC 147 by Kraus et al. 29]. The model computed for MWC 147 assumes a spherical shell geometry. Panels (a) and (b) show the dust density and the temperature distribution. Panels (c) and (d) show the ray-traced images for two representative NIR $(2.25 \mu \mathrm{m})$ and MIR $(10.0 \mu \mathrm{m})$ wavelengths. Panel (e) shows the SED for various inclination angles, whereas panel (f) gives the SED for the best-fit inclination angle and separates the flux which originates in stellar photospheric emission, thermal emission, dust irradiation, and accretion luminosity. Finally, panels (g) to (j) depict the NIR and MIR visibilities computed from their radiative transfer models. 
SED and the visibilities of RY Tau as mentioned earlier with 2 different models although several other models have been dismissed with MIDI data. The region where the measurements would allow us to choose between the two remaining models is located inward, and can be observed at shorter wavelengths.

\subsection{Dust mineralogy}

The mid-infrared wavelength region contains strong resonances of abundant dust species, both oxygen-rich (amorphous or crystalline silicates) and carbon-rich (polycyclic aromatic hydrocarbons, or PAHs). Therefore, spectroscopy of optically thick protoplanetary disks offers a diagnostic of the chemical composition and grain size of dust in disk atmospheres.

van Boekel et al. 61] spatially resolved three protoplanetary disks surrounding Herbig Ae/Be stars across the $N$ band. The correlated spectra measured by MIDI at the VLTI correspond to disk regions ranging from 1 to 2 AUs. By combining these measurements with unresolved spectra, the spectrum corresponding to outer disk regions at $220 \mathrm{AU}$ can also be derived. These observations have revealed that the dust in these regions was highly crystallized (40 to 100\%), more than any other dust observed in young stars until now. The spectral shape of the inner-disk spectra shows surprising similarity with Solar System comets. Their observations imply that silicates crystallize before terrestrial planets are formed, consistent with the composition of meteorites in the Solar System. Similar measurements were also carried out by Ratzka et al. [51] on the T Tauri system, TW Hya. According to the correlated flux measured with MIDI, most of the crystalline material is located in the inner, unresolved part of the disk, about $1 \mathrm{AU}$ in radius.

\subsection{Gas/dust connection}

Gil et al. [23] observed the young stellar system 51 Oph confirming the interpretation of Thi et al. [59] and more recently Berthoud et al. [8] of a disk seen nearly edge-on: the radial distribution of excitation temperatures for the vibrational levels of CO overtone $(\Delta v=2)$ emission from hot gas is consistent with the gas being in radiative thermal equilibrium except at the inner edge, where low vibrational bands have higher excitation temperatures. Tatulli et al. 58 confirmed the high inclination of the disk but also detect the CO bandheads allowing the dust responsible for the continuum to be separated from the gas. As a matter of fact, the visibilities in the $\mathrm{CO}$ bands is lower than the ones measured in the continuum implying that the region responsible for this gas emission is smaller than the region responsible for the dust emission. Figure 5 illustrates this result, and shows that the combination of very high spatial information with spectral resolution opens brand new perspectives in the studies of the inner disk properties by discriminating between components.

\section{Other AU-scale phenomena}

Several other physical phenomena have been investigated in the innermost region of disks: wind, magnetosphere and close companions. 


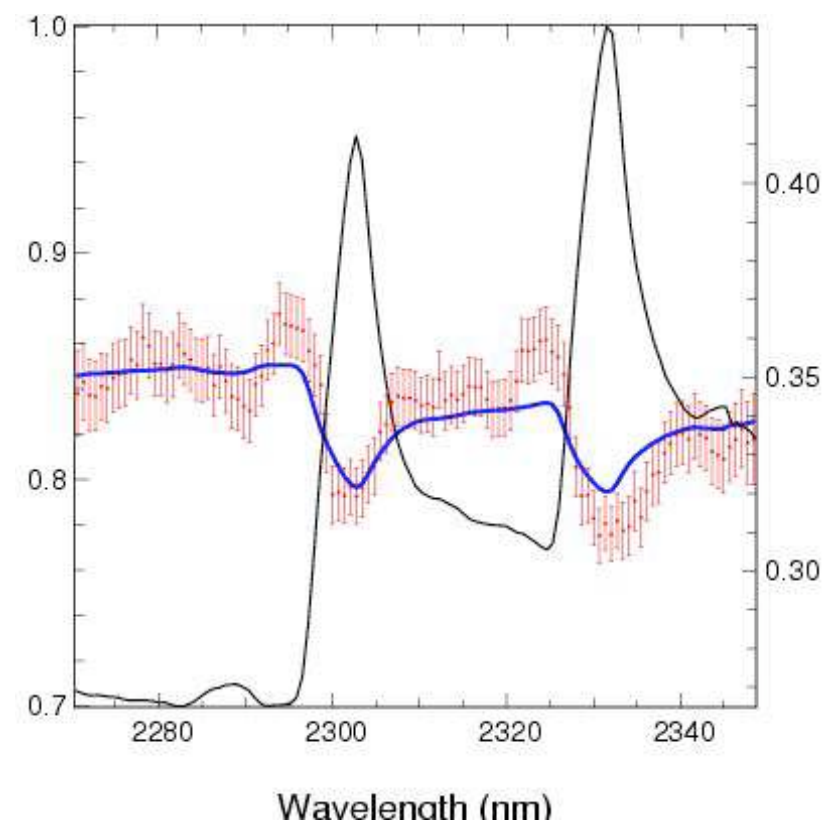

Figure 5: Spectrally dispersed visibility amplitudes of 51 Oph in the CO bandhead spectral region. Overimposed is the spectrum as measured by VLTI/AMBER (black line). The blue curve corresponds to the addition of a simple uniform disk model for the excess emission in the line with a typical diameter of $0.2 \mathrm{AU}$. From Tatulli et al. (priv. comm.)

\subsection{Outflows and winds}

The power of spectrally resolved interferometric measurements provides detailed wavelength dependence of inner disk continuum emission (see end of Sect. 3.2). These new capabilities enable also detailed studies of hot winds and outflows, and therefore the physical conditions and kinematics of the gaseous components in which emission and absorption lines arise like $\mathrm{Br} \gamma$ and $\mathrm{H}_{2}$ ones. With VLTI/AMBER, Malbet et al. [35] spatially resolved the luminous Herbig Be object MWC 297, measuring visibility amplitudes as a function of wavelength at intermediate spectral resolution $\mathrm{R}=1500$ across a $2.0-2.2 \mu \mathrm{m}$ band, and in particular the $\operatorname{Br} \gamma$ emission line. The interferometer visibilities in the $\operatorname{Br} \gamma$ line are about $30 \%$ lower than those of the nearby continuum, showing that the Br $\gamma$ emitting region is significantly larger than the NIR continuum region. Known to be an outflow source, a preliminary model has been constructed in which a gas envelope, responsible for the $\operatorname{Br} \gamma$ emission, surrounds an optically thick circumstellar disk. The characteristic size of the line-emitting region being $40 \%$ larger than that of the NIR disk. This model is successful at reproducing the VLTI/AMBER measurements as well as previous continuum interferometric measurements at shorter and longer baselines [42, 21], the SED, and the shapes of the $\mathrm{H} \alpha, \mathrm{H} \beta$, and $\mathrm{Br} \gamma$ emission lines. The precise nature of the MWC 297 wind, however, remains unclear; the limited amount of data obtained in these first observations cannot, for example, discriminate between a stellar or disk origin for the wind, or between competing models of disk winds [e.g. [54, 22]. 


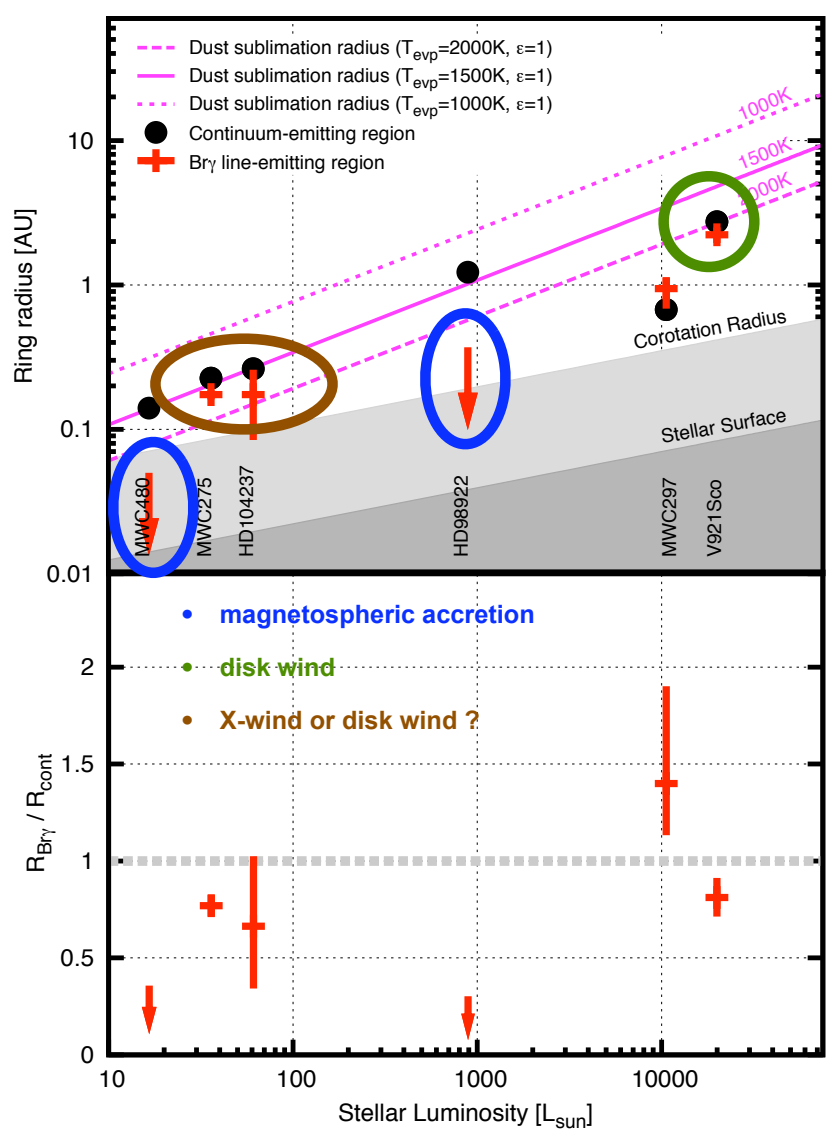

Figure 6: A systematic study of the origin of the $\mathrm{Br}_{\gamma}$ emission in Herbig Ae/Be stars from Boden et al. [10]. Top: the fitted ring radii for the continuum (black points) and $\mathrm{Br}_{\gamma}$ line (red points) plotted as a function of stellar luminosity. The spatial extension of the stellar surface is represented in grey and the dust sublimation radius corresponding to the dust sublimation temperatures of 2000, 1500, and $1000 \mathrm{~K}$. Bottom: the ratio of normalized size of the $\mathrm{Br}_{\gamma}$ region by the size of the continuum-emitting region. Circles in blue emphasize the system for which a magnetospheric accretion scenario is a compatible model, in green the ones with probable disk winds and in brown the ones with $\mathrm{X}$ wind or disk wind.

\subsection{Magnetosphere}

The origin of the hydrogen line emission in Herbig Ae/Be stars is still unclear. The lines may originate either in the gas which accretes onto the star from the disk, as in magnetospheric accretion models [24], or in winds and jets, driven by the interaction of the accreting disk with a stellar [53] or disk [11] magnetic field. For all models, emission in the hydrogen lines is predicted to occur over very small spatial scales, a few AUs at most. To understand the physical processes that happen at these scales, one needs to combine very high spatial resolution with enough spectral resolution to resolve the line profile.

On the one hand, Tatulli et al. [57] performed interferometric observations of the Herbig Ae star HD 104237, obtained with the VLTI/AMBER instrument with $R=1500$ high spectral resolution. The observed visibility was identical in the $\mathrm{Br} \gamma$ line and in the continuum, even though the line represents $35 \%$ of the continuum flux. This immediately implies that 
the line and continuum emission regions have the same apparent size. Using simple models to describe the $\operatorname{Br} \gamma$ emission, they showed that the line emission is unlikely to originate in either magnetospheric accreting columns of gas or in the gaseous disk but more likely in a compact outflowing disk wind launched in the vicinity of the rim, about $0.5 \mathrm{AU}$ from the star. The main part of the $\mathrm{Br} \gamma$ emission in HD 104237 is unlikely to originate in magnetospheric accreting matter.

On the other hand, Eisner [15] measured an increase of the $\operatorname{Br} \gamma$ visibility in MWC 480 implying that the region of emission of the hydrogen line is very compact, less than 0.1 mas in radius which could be interpreted as emission originating in the magnetosphere of the system.

At the present time, given the limited number of samples, it is difficult to derive a general tendency but it seems that all possible scenari can be found like Kraus et al. [28] show in a mini-survey of five Herbig Ae/Be stars.

\subsection{Binaries and multiple systems}

Boden et al. 9] performed the first direct measurement of pre-main sequence stellar masses using interferometry, for the double-lined system HD 98800-B. These authors established a preliminary orbit that allowed determination of the (subsolar) masses of the individual components with $8 \%$ accuracy. Comparison with stellar models indicates the need for subsolar abundances for both components, although stringent tests of competing models will only become possible when more observations improve the orbital phase coverage and thus the accuracy of the stellar masses derived. Boden et al. [10] published another determination of dynamical masses for the pre-mains sequence system V773 Tau A.

Another example, based on a low-level oscillation in the visibility amplitude signature in the PTI data of FU Ori, Malbet et al. [38] claimed the detection of an off-centered spot embedded in the disk that could be physically interpreted as a young stellar or protoplanetary companion located at $\sim 10 \mathrm{AUs}$, and could possibly be at the origin of the FU Ori outburst itself. Using another technique, Millan-Gabet et al. 41] reported on the detection of localized off-center emission at 1-4 AU in the circumstellar environment of AB Aurigae. They used closure-phase measurements in the near-infrared. When probing sub-AU scales, all closure phases are close to zero degrees, as expected given the previously determined size of the AB Aurigae inner-dust disk. However, a clear closure-phase signal of $-3.5^{\circ} \pm 0.5^{\circ}$ is detected on one triangle containing relatively short baselines, requiring a high degree of asymmetry from emission at larger AU scales in the disk. They interpret such detected asymmetric nearinfrared emission as a result of localized viscous heating due to a gravitational instability in the AB Aurigae disk, or to the presence of a close stellar companion or accreting substellar object.

\section{Future prospects and conclusion}

As emphasized in this review, more interferometric data is required with better accuracy and also wider coverage of the baselines in order to constrain better the models that have been proposed. Like for radio astronomy, these supplementary data will allow image 
reconstruction without any prior knowledge of the observed structure. Several facilities are already ready to obtain interferometric images although with few pixels across the field: MIRC at CHARA and AMBER at the VLTI in the near-infrared. However at the moment MIRC is limited in sensitivity and AMBER in number of telescopes (3) which makes it difficult to routinely image. In the mid-infrared the MATISSE instrument has been proposed to ESO to provide imaging with 4 telecopes at the VLTI. VSI is also a proposed second generation VLTI instrument which will be able to combine from 4 to 8 beams at the same time so that imaging becomes easier. The LBT will also provide imaging capability.

All these instruments provide spectral resolution that make them indeed spectro-imagers. Therefore in the future, one should be able to obtain a wealth of information from the innermost regions of disks around young stars. However in the meantime, observations are already mature enough to allow detailed modeling of the phenomena occuring in these inner regions of young stellar objects.

Acknowledgements. The author is grateful to Willem-Jan de Wit for a careful reading of the manuscript and his fruitful comments.

\section{References}

[1] Ábrahám, P., Mosoni, L., Henning, T., Kóspál, Á., Leinert, C., Quanz, S. P., Ratzka, T., Apr. 2006. First AU-scale observations of V1647 Orionis with VLTI/MIDI. A\&A449, L13-L16.

[2] Acke, B., Verhoelst, T., van den Ancker, M. E., Deroo, P., Waelkens, C., Chesneau, O., Tatulli, E., Benisty, M., Puga, E., Waters, L. B. F. M., Verhoeff, A., de Koter, A., Jul. 2008. MWC 297: a young high-mass star rotating at critical velocity. $A \mathscr{E} A 485,209-221$.

[3] Akeson, R., Jun. 2008. Observations of circumstellar disks with infrared interferometry. New Astronomy Review 52, 94-104.

[4] Akeson, R. L., Oct. 2008. Observations of circumstellar disks with infrared interferometry. Journal of Physics Conference Series 131 (1), 012019-+.

[5] Akeson, R. L., Boden, A. F., Monnier, J. D., Millan-Gabet, R., Beichman, C., Beletic, J., Calvet, N., Hartmann, L., Hillenbrand, L., Koresko, C., Sargent, A., Tannirkulam, A., Dec. 2005. Keck Interferometer Observations of Classical and Weak-line T Tauri Stars. ApJ635, 1173-1181.

[6] Akeson, R. L., Ciardi, D. R., van Belle, G. T., Creech-Eakman, M. J., Lada, E. A., Nov. 2000. Infrared Interferometric Observations of Young Stellar Objects. ApJ543, 313-317.

[7] Akeson, R. L., Walker, C. H., Wood, K., Eisner, J. A., Scire, E., Penprase, B., Ciardi, D. R., van Belle, G. T., Whitney, B., Bjorkman, J. E., Mar. 2005. Observations and Modeling of the Inner Disk Region of T Tauri Stars. ApJ622, 440-450.

[8] Berthoud, M. G., Keller, L. D., Herter, T. L., Richter, M. J., Whelan, D. G., May 2007. Near-IR CO Overtone Emission in 51 Ophiuchi. ApJ660, 461-468.

[9] Boden, A. F., Sargent, A. I., Akeson, R. L., Carpenter, J. M., Torres, G., Latham, D. W., Soderblom, D. R., Nelan, E., Franz, O. G., Wasserman, L. H., Dec. 2005. Dynamical Masses for Low-Mass PreMain-Sequence Stars: A Preliminary Physical Orbit for HD 98800 B. ApJ635, 442-451.

[10] Boden, A. F., Torres, G., Sargent, A. I., Akeson, R. L., Carpenter, J. M., Boboltz, D. A., Massi, M., Ghez, A. M., Latham, D. W., Johnston, K. J., Menten, K. M., Ros, E., Dec. 2007. Dynamical Masses for Pre-Main-Sequence Stars: A Preliminary Physical Orbit for V773 Tau A. ApJ670, 1214-1224.

[11] Casse, F., Ferreira, J., Jan. 2000. Magnetized accretion-ejection structures. IV. Magnetically-driven jets from resistive, viscous, Keplerian discs. A\&A353, 1115-1128.

[12] Colavita, M., Akeson, R., Wizinowich, P., Shao, M., Acton, S., Beletic, J., Bell, J., Berlin, J., Boden, A., Booth, A., Boutell, R., Chaffee, F., Chan, D., Chock, J., Cohen, R., Crawford, S., Creech-Eakman, M., Eychaner, G., Felizardo, C., Gathright, J., Hardy, G., Henderson, H., Herstein, J., Hess, M., Hovland, 
E., Hrynevych, M., Johnson, R., Kelley, J., Kendrick, R., Koresko, C., Kurpis, P., Le Mignant, D., Lewis, H., Ligon, E., Lupton, W., McBride, D., Mennesson, B., Millan-Gabet, R., Monnier, J., Moore, J., Nance, C., Neyman, C., Niessner, A., Palmer, D., Reder, L., Rudeen, A., Saloga, T., Sargent, A., Serabyn, E., Smythe, R., Stomski, P., Summers, K., Swain, M., Swanson, P., Thompson, R., Tsubota, K., Tumminello, A., van Belle, G., Vasisht, G., Vause, J., Walker, J., Wallace, K., Wehmeier, U., Aug. 2003. Observations of DG Tauri with the Keck Interferometer. ApJ592, L83-L86.

[13] de Wit, W. J., Hoare, M. G., Oudmaijer, R. D., Mottram, J. C., Dec. 2007. VLTI/MIDI $10 \mu \mathrm{m}$ Interferometry of the Forming Massive Star W33A. ApJ671, L169-L172.

[14] Dullemond, C. P., Dominik, C., Natta, A., Oct. 2001. Passive Irradiated Circumstellar Disks with an Inner Hole. ApJ560, 957-969.

[15] Eisner, J. A., May 2007. Water vapour and hydrogen in the terrestrial-planet-forming region of a protoplanetary disk. Nature $447,562-564$.

[16] Eisner, J. A., Chiang, E. I., Hillenbrand, L. A., Feb. 2006. Spatially Resolving the Inner Disk of TW Hydrae. ApJ637, L133-L136.

[17] Eisner, J. A., Chiang, E. I., Lane, B. F., Akeson, R. L., Mar. 2007. Spectrally Dispersed K-Band Interferometric Observations of Herbig Ae/Be Sources: Inner Disk Temperature Profiles. ApJ657, 347358.

[18] Eisner, J. A., Hillenbrand, L. A., White, R. J., Akeson, R. L., Sargent, A. I., Apr. 2005. Observations of T Tauri Disks at Sub-AU Radii: Implications for Magnetospheric Accretion and Planet Formation. ApJ623, 952-966.

[19] Eisner, J. A., Hillenbrand, L. A., White, R. J., Bloom, J. S., Akeson, R. L., Blake, C. H., Nov. 2007. Near-Infrared Interferometric, Spectroscopic, and Photometric Monitoring of T Tauri Inner Disks. ApJ669, 1072-1084.

[20] Eisner, J. A., Lane, B. F., Akeson, R. L., Hillenbrand, L. A., Sargent, A. I., May 2003. Near-Infrared Interferometric Measurements of Herbig Ae/Be Stars. ApJ588, 360-372.

[21] Eisner, J. A., Lane, B. F., Hillenbrand, L. A., Akeson, R. L., Sargent, A. I., Oct. 2004. Resolved Inner Disks around Herbig Ae/Be Stars. ApJ613, 1049-1071.

[22] Ferreira, J., Bessolaz, N., Zanni, C., Combet, C., May 2007. Large scale magnetic fields in discs: jets and reconnection X-winds. In: Bouvier, J., Appenzeller, I. (Eds.), IAU Symposium. Vol. 243 of IAU Symposium. pp. 307-314.

[23] Gil, C., Malbet, F., Schöller, M., Chesneau, O., Leinert, C., 2008. Observations of 51 Ophiuchi with MIDI at the VLTI. In: Richichi, A., Delplancke, F., Paresce, F., Chelli, A. (Eds.), The Power of Optical/IR Interferometry: Recent Scientific Results and 2nd Generation. pp. 187-+.

[24] Hartmann, L., Hewett, R., Calvet, N., May 1994. Magnetospheric accretion models for T Tauri stars. 1: Balmer line profiles without rotation. ApJ426, 669-687.

[25] Isella, A., Natta, A., Aug. 2005. The shape of the inner rim in proto-planetary disks. A $3 A 438,899-907$.

[26] Isella, A., Tatulli, E., Natta, A., Testi, L., May 2008. Gas and dust in the inner disk of the Herbig Ae star MWC 758. A $3 A 483$, L13-L16.

[27] Kraus, S., Balega, Y. Y., Berger, J.-P., Hofmann, K.-H., Millan-Gabet, R., Monnier, J. D., Ohnaka, K., Pedretti, E., Preibisch, T., Schertl, D., Schloerb, F. P., Traub, W. A., Weigelt, G., May 2007. Visual/infrared interferometry of Orion Trapezium stars: preliminary dynamical orbit and aperture synthesis imaging of the $\theta^{1}$ Orionis C system. A\&A466, 649-659.

[28] Kraus, S., Hofmann, K.-H., Benisty, M., Berger, J.-P., Chesneau, O., Isella, A., Malbet, F., Meilland, A., Nardetto, N., Natta, A., Preibisch, T., Schertl, D., Smith, M., Stee, P., Tatulli, E., Testi, L., Weigelt, G., Oct. 2008. The origin of hydrogen line emission for five Herbig Ae/Be stars spatially resolved by VLTI/AMBER spectro-interferometry. A\&A489, 1157-1173.

[29] Kraus, S., Preibisch, T., Ohnaka, K., Mar. 2008. Detection of an Inner Gaseous Component in a Herbig Be Star Accretion Disk: Near- and Mid-Infrared Spectrointerferometry and Radiative Transfer modeling of MWC 147. ApJ676, 490-508.

[30] Lachaume, R., Malbet, F., Monin, J.-L., Mar. 2003. The vertical structure of T Tauri accretion discs. III. Consistent interpretation of spectra and visibilities with a two-layer model. AESA400, 185-202. 
[31] Lachaume, R., Preibisch, T., Driebe, T., Weigelt, G., Jul. 2007. Resolving the B[e] star Hen 3-1191 at $10 \mu \mathrm{m}$ with VLTI/MIDI. A $\& A 469,587-593$.

[32] Leinert, C., van Boekel, R., Waters, L. B. F. M., Chesneau, O., Malbet, F., Köhler, R., Jaffe, W., Ratzka, T., Dutrey, A., Preibisch, T., Graser, U., Bakker, E., Chagnon, G., Cotton, W. D., Dominik, C., Dullemond, C. P., Glazenborg-Kluttig, A. W., Glindemann, A., Henning, T., Hofmann, K.-H., de Jong, J., Lenzen, R., Ligori, S., Lopez, B., Meisner, J., Morel, S., Paresce, F., Pel, J.-W., Percheron, I., Perrin, G., Przygodda, F., Richichi, A., Schöller, M., Schuller, P., Stecklum, B., van den Ancker, M. E., von der Lühe, O., Weigelt, G., Aug. 2004. Mid-infrared sizes of circumstellar disks around Herbig Ae/Be stars measured with MIDI on the VLTI. A\&A423, 537-548.

[33] Li Causi, G., Antoniucci, S., Tatulli, E., Feb. 2008. De-biasing interferometric visibilities in VLTIAMBER data of low SNR observations. A\&A479, 589-595.

[34] Malbet, F., May 2007. Inner disk regions revealed by infrared interferometry. In: Bouvier, J., Appenzeller, I. (Eds.), IAU Symposium. Vol. 243 of IAU Symposium. pp. 123-134.

[35] Malbet, F., Benisty, M., de Wit, W.-J., Kraus, S., Meilland, A., Millour, F., Tatulli, E., Berger, J.-P., Chesneau, O., Hofmann, K.-H., Isella, A., Natta, A., Petrov, R. G., Preibisch, T., Stee, P., Testi, L., Weigelt, G., Antonelli, P., Beckmann, U., Bresson, Y., Chelli, A., Dugué, M., Duvert, G., Gennari, S., Glück, L., Kern, P., Lagarde, S., Le Coarer, E., Lisi, F., Perraut, K., Puget, P., Rantakyrö, F., Robbe-Dubois, S., Roussel, A., Zins, G., Accardo, M., Acke, B., Agabi, K., Altariba, E., Arezki, B., Aristidi, E., Baffa, C., Behrend, J., Blöcker, T., Bonhomme, S., Busoni, S., Cassaing, F., Clausse, J.M., Colin, J., Connot, C., Delboulbé, A., Domiciano de Souza, A., Driebe, T., Feautrier, P., Ferruzzi, D., Forveille, T., Fossat, E., Foy, R., Fraix-Burnet, D., Gallardo, A., Giani, E., Gil, C., Glentzlin, A., Heiden, M., Heininger, M., Hernandez Utrera, O., Kamm, D., Kiekebusch, M., Le Contel, D., Le Contel, J.-M., Lesourd, T., Lopez, B., Lopez, M., Magnard, Y., Marconi, A., Mars, G., Martinot-Lagarde, G., Mathias, P., Mège, P., Monin, J.-L., Mouillet, D., Mourard, D., Nussbaum, E., Ohnaka, K., Pacheco, J., Perrier, C., Rabbia, Y., Rebattu, S., Reynaud, F., Richichi, A., Robini, A., Sacchettini, M., Schertl, D., Schöller, M., Solscheid, W., Spang, A., Stefanini, P., Tallon, M., Tallon-Bosc, I., Tasso, D., Vakili, F., von der Lühe, O., Valtier, J.-C., Vannier, M., Ventura, N., Mar. 2007. Disk and wind interaction in

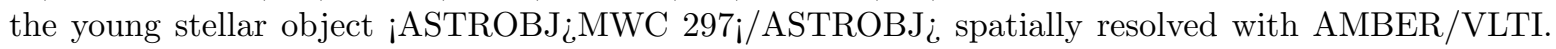
A 8 A464, 43-53.

[36] Malbet, F., Berger, J.-P., Colavita, M. M., Koresko, C. D., Beichman, C., Boden, A. F., Kulkarni, S. R., Lane, B. F., Mobley, D. W., Pan, X. P., Shao, M., van Belle, G. T., Wallace, J. K., Nov. 1998. FU Orionis Resolved by Infrared Long-Baseline Interferometry at a 2 AU Scale. ApJ507, L149-L152.

[37] Malbet, F., Bertout, C., Oct. 1995. Detecting T Tauri disks with optical long-baseline interferometry. A\&AS113, 369-+.

[38] Malbet, F., Lachaume, R., Berger, J.-P., Colavita, M. M., di Folco, E., Eisner, J. A., Lane, B. F., Millan-Gabet, R., Ségransan, D., Traub, W. A., Jul. 2005. New insights on the AU-scale circumstellar structure of FU Orionis. A\& $A 437,627-636$.

[39] Millan-Gabet, R., Malbet, F., Akeson, R., Leinert, C., Monnier, J., Waters, R., 2007. The Circumstellar Environments of Young Stars at AU Scales. In: Reipurth, B., Jewitt, D., Keil, K. (Eds.), Protostars and Planets V. pp. 539-554.

[40] Millan-Gabet, R., Monnier, J. D., Akeson, R. L., Hartmann, L., Berger, J.-P., Tannirkulam, A., Melnikov, S., Billmeier, R., Calvet, N., D'Alessio, P., Hillenbrand, L. A., Kuchner, M., Traub, W. A., Tuthill, P. G., Beichman, C., Boden, A., Booth, A., Colavita, M., Creech-Eakman, M., Gathright, J., Hrynevych, M., Koresko, C., Le Mignant, D., Ligon, R., Mennesson, B., Neyman, C., Sargent, A., Shao, M., Swain, M., Thompson, R., Unwin, S., van Belle, G., Vasisht, G., Wizinowich, P., Apr. 2006. Keck Interferometer Observations of FU Orionis Objects. ApJ641, 547-555.

[41] Millan-Gabet, R., Monnier, J. D., Berger, J.-P., Traub, W. A., Schloerb, F. P., Pedretti, E., Benisty, M., Carleton, N. P., Haguenauer, P., Kern, P., Labeye, P., Lacasse, M. G., Malbet, F., Perraut, K., Pearlman, M., Thureau, N., Jul. 2006. Bright Localized Near-Infrared Emission at 1-4 AU in the AB Aurigae Disk Revealed by IOTA Closure Phases. ApJ645, L77-L80.

[42] Millan-Gabet, R., Schloerb, F. P., Traub, W. A., Jan. 2001. Spatially Resolved Circumstellar Structure 
of Herbig AE/BE Stars in the Near-Infrared. ApJ546, 358-381.

[43] Millan-Gabet, R., Schloerb, F. P., Traub, W. A., Malbet, F., Berger, J. P., Bregman, J. D., Mar. 1999. Sub-Astronomical Unit Structure of the Near-Infrared Emission from AB Aurigae. ApJ513, L131-L134.

[44] Monnier, J. D., Berger, J.-P., Millan-Gabet, R., Traub, W. A., Schloerb, F. P., Pedretti, E., Benisty, M., Carleton, N. P., Haguenauer, P., Kern, P., Labeye, P., Lacasse, M. G., Malbet, F., Perraut, K., Pearlman, M., Zhao, M., Aug. 2006. Few Skewed Disks Found in First Closure-Phase Survey of Herbig Ae/Be Stars. ApJ647, 444-463.

[45] Monnier, J. D., Millan-Gabet, R., Nov. 2002. On the Interferometric Sizes of Young Stellar Objects. ApJ579, 694-698.

[46] Monnier, J. D., Millan-Gabet, R., Billmeier, R., Akeson, R. L., Wallace, D., Berger, J.-P., Calvet, N., D’Alessio, P., Danchi, W. C., Hartmann, L., Hillenbrand, L. A., Kuchner, M., Rajagopal, J., Traub, W. A., Tuthill, P. G., Boden, A., Booth, A., Colavita, M., Gathright, J., Hrynevych, M., Le Mignant, D., Ligon, R., Neyman, C., Swain, M., Thompson, R., Vasisht, G., Wizinowich, P., Beichman, C., Beletic, J., Creech-Eakman, M., Koresko, C., Sargent, A., Shao, M., van Belle, G., May 2005. The Near-Infrared Size-Luminosity Relations for Herbig Ae/Be Disks. ApJ624, 832-840.

[47] Muzerolle, J., D’Alessio, P., Calvet, N., Hartmann, L., Dec. 2004. Magnetospheres and Disk Accretion in Herbig Ae/Be Stars. ApJ617, 406-417.

[48] Natta, A., Prusti, T., Neri, R., Wooden, D., Grinin, V. P., Mannings, V., May 2001. A reconsideration of disk properties in Herbig Ae stars. AछA371, 186-197.

[49] Preibisch, T., Kraus, S., Driebe, T., van Boekel, R., Weigelt, G., Oct. 2006. A compact dusty disk around the Herbig Ae star HR 5999 resolved with VLTI / MIDI. AEAA458, 235-243.

[50] Quanz, S. P., Henning, T., Bouwman, J., Ratzka, T., Leinert, C., Sep. 2006. FU Orionis: The MIDI VLTI Perspective. ApJ648, 472-483.

[51] Ratzka, T., Leinert, C., Henning, T., Bouwman, J., Dullemond, C. P., Jaffe, W., Aug. 2007. High spatial resolution mid-infrared observations of the low-mass young star TW Hydrae. A\&A471, 173-185.

[52] Schegerer, A. A., Wolf, S., Ratzka, T., Leinert, C., Feb. 2008. The T Tauri star RY Tauri as a case study of the inner regions of circumstellar dust disks. A $64478,779-793$.

[53] Shu, F., Najita, J., Ostriker, E., Wilkin, F., Ruden, S., Lizano, S., Jul. 1994. Magnetocentrifugally driven flows from young stars and disks. 1: A generalized model. ApJ429, 781-796.

[54] Shu, F. H., Galli, D., Lizano, S., Cai, M. J., May 2007. Magnetization, accretion, and outflows in young stellar objects. In: Bouvier, J., Appenzeller, I. (Eds.), IAU Symposium. Vol. 243 of IAU Symposium. pp. 249-264.

[55] Tannirkulam, A., Harries, T. J., Monnier, J. D., May 2007. The Inner Rim of YSO Disks: Effects of Dust Grain Evolution. ApJ661, 374-384.

[56] Tannirkulam, A., Monnier, J. D., Millan-Gabet, R., Harries, T. J., Pedretti, E., ten Brummelaar, T. A., McAlister, H., Turner, N., Sturmann, J., Sturmann, L., Apr. 2008. Strong Near-Infrared Emission Interior to the Dust Sublimation Radius of Young Stellar Objects MWC 275 and AB Aurigae. ApJ677, L51-L54.

[57] Tatulli, E., Isella, A., Natta, A., Testi, L., Marconi, A., Malbet, F., Stee, P., Petrov, R. G., Millour, F., Chelli, A., Duvert, G., Antonelli, P., Beckmann, U., Bresson, Y., Dugué, M., Gennari, S., Glück, L., Kern, P., Lagarde, S., Le Coarer, E., Lisi, F., Perraut, K., Puget, P., Rantakyrö, F., Robbe-Dubois, S., Roussel, A., Weigelt, G., Zins, G., Accardo, M., Acke, B., Agabi, K., Altariba, E., Arezki, B., Aristidi, E., Baffa, C., Behrend, J., Blöcker, T., Bonhomme, S., Busoni, S., Cassaing, F., Clausse, J.-M., Colin, J., Connot, C., Delboulbé, A., Domiciano de Souza, A., Driebe, T., Feautrier, P., Ferruzzi, D., Forveille, T., Fossat, E., Foy, R., Fraix-Burnet, D., Gallardo, A., Giani, E., Gil, C., Glentzlin, A., Heiden, M., Heininger, M., Hernandez Utrera, O., Hofmann, K.-H., Kamm, D., Kiekebusch, M., Kraus, S., Le Contel, D., Le Contel, J.-M., Lesourd, T., Lopez, B., Lopez, M., Magnard, Y., Mars, G., MartinotLagarde, G., Mathias, P., Mège, P., Monin, J.-L., Mouillet, D., Mourard, D., Nussbaum, E., Ohnaka, K., Pacheco, J., Perrier, C., Rabbia, Y., Rebattu, S., Reynaud, F., Richichi, A., Robini, A., Sacchettini, M., Schertl, D., Schöller, M., Solscheid, W., Spang, A., Stefanini, P., Tallon, M., Tallon-Bosc, I., Tasso, D., Vakili, F., von der Lühe, O., Valtier, J.-C., Vannier, M., Ventura, N., Mar. 2007. Constraining the 
wind launching region in Herbig Ae stars: AMBER/VLTI spectroscopy of HD 104237. A\& A464, 55-58.

[58] Tatulli, E., Malbet, F., Ménard, F., Gil, C., Testi, L., Natta, A., Kraus, S., Stee, P., Robbe-Dubois, S., Oct. 2008. Spatially resolving the hot CO around the young Be star 51 Ophiuchi. A $8 A 489,1151-1155$.

[59] Thi, W.-F., van Dalen, B., Bik, A., Waters, L. B. F. M., Jan. 2005. Evidence for a hot dust-free inner disk around 51 Oph. A 6 A430, L61-L64.

[60] Tuthill, P. G., Monnier, J. D., Danchi, W. C., Hale, D. D. S., Townes, C. H., Oct. 2002. Imaging the Disk around the Luminous Young Star $\mathrm{LkH} \alpha 101$ with Infrared Interferometry. ApJ577, 826-838.

[61] van Boekel, R., Min, M., Leinert, C., Waters, L. B. F. M., Richichi, A., Chesneau, O., Dominik, C., Jaffe, W., Dutrey, A., Graser, U., Henning, T., de Jong, J., Köhler, R., de Koter, A., Lopez, B., Malbet, F., Morel, S., Paresce, F., Perrin, G., Preibisch, T., Przygodda, F., Schöller, M., Wittkowski, M., Nov. 2004. The building blocks of planets within the 'terrestrial' region of protoplanetary disks. Nature 432 , 479-482.

[62] Vinković, D., Ivezić, Ž., Miroshnichenko, A. S., Elitzur, M., Dec. 2003. Discs and haloes in pre-mainsequence stars. MNRAS346, 1151-1161.

[63] Vinković, D., Jurkić, T., Mar. 2007. Relation between the Luminosity of Young Stellar Objects and Their Circumstellar Environment. ApJ658, 462-479.

[64] Wilkin, F. P., Akeson, R. L., 2003. Palomar Testbed Interferometer Observations of Young Stellar Objects. Ap\&SSS286, 145-150.

[65] Zhu, Z., Hartmann, L., Calvet, N., Hernandez, J., Tannirkulam, A.-K., D’Alessio, P., Sep. 2008. LongWavelength Excesses of FU Orionis Objects: Flared Outer Disks or Infalling Envelopes? ApJ684, 1281-1290. 